\title{
Sporadic E layer at mid-latitudes: average properties and influence of atmospheric tides
}

\author{
A. Pignalberi ${ }^{1}$, M. Pezzopane ${ }^{2}$, and E. Zuccheretti ${ }^{2}$ \\ ${ }^{1}$ Dipartimento di Fisica, Università di Roma "La Sapienza", 00185, Rome, Italy \\ ${ }^{2}$ Istituto Nazionale di Geofisica e Vulcanologia, 00143, Rome, Italy \\ Correspondence to: M. Pezzopane (michael.pezzopane@ingv.it)
}

Received: 30 July 2014 - Revised: 6 October 2014 - Accepted: 9 October 2014 - Published: 21 November 2014

\begin{abstract}
This paper describes a study of the daily variability shown by the main characteristics of the sporadic $\mathrm{E}$ (Es) layer, that is the top frequency (ftEs) and the lowest virtual height (h'Es). The study is based on ionograms recorded by the Advanced Ionospheric Sounder by the Istituto Nazionale di Geofisica e Vulcanologia (AIS-INGV) ionosondes installed in the ionospheric stations at Rome $\left(41.8^{\circ} \mathrm{N}, 12.5^{\circ} \mathrm{E}\right)$ and Gibilmanna $\left(37.9^{\circ} \mathrm{N}, 14.0^{\circ} \mathrm{E}\right)$, Italy, during the summer (June, July, August and September) of 2013, a year falling in the ascending phase of solar cycle 24 . The ftEs presents a diurnal variation characterized by two maxima, the first around noon is very well defined and the second in the evening/night is much less defined; the amplitude of both maxima decreases from June to September accompanied by a general decrease of the ftEs values which is more pronounced in the daytime than in the nighttime. h'Es also presents a diurnal variation characterized by two maxima but, unlike ftEs, these present the same amplitude which is independent from the considered month. Assuming that both ftEs and h'Es trends are influenced by the atmospheric tides, the height-time-intensity (HTI) technique was applied to deeply investigate how these waves control the Es dynamics. The HTI study, along with a fast Fourier transform analysis, show that a well-defined semidiurnal periodicity characterizes the Es layer dynamics most accurately in June and July, while in August and September the daytime semidiurnal periodicity becomes weaker and the role of the diurnal periodicity is consequently highlighted.
\end{abstract}

Keywords. Ionosphere (ionosphere-atmosphere interactions; mid-latitude ionosphere; instruments and techniques)

\section{Introduction}

The term sporadic E (Es) layer refers to extremely anomalous values of ionization confined within layers of very limited thickness (a few km) occurring between 90 and $130 \mathrm{~km}$, whose critical frequency is often higher than the normal $\mathrm{E}$ layer. Es layers have been widely studied from the early twentieth century (e.g., see reviews by Whitehead, 1989; Mathews, 1998, and references therein), because they can seriously affect high frequency (HF) radio communications. These layers form in the ionospheric dynamo region, when metallic ions of meteoric origin converge vertically, mainly due to vertical shears in the horizontal wind (e.g., see reviews by Haldoupis, 2011, 2012, and references therein).

The periodic heating of the atmosphere by the Sun generates tides whose periods are related to the solar day. It might initially be estimated that the diurnal solar heating would give rise to tides with a period of $24 \mathrm{~h}$, corresponding to the heating periodicity. However, observations reveal that large amplitude tides are generated with periods of 24, 12, 8 and $6 \mathrm{~h}$, even though these latter tides are characterized by smaller amplitudes. These periods appear because the solar heating of the atmosphere occurs in an approximate square wave profile and is consequently rich in harmonics (Forbes, 1995).

With regard to this, it is now known that diurnal and semidiurnal tides play a significant role in affecting the development and descent of Es layers and, given that the prevailing winds in the $\mathrm{E}$ region are thermal tides generated by solar heating, this is not unexpected (see, e.g., Lindzen and Chapman, 1969; Forbes, 1995). However, it is worth highlighting that the wind-shear theory (perhaps with a small added external field) appears sufficient, along with the tidal wind system, 
to explain the most common mid-latitude Es layers that are known as tidal ion layers (TILs). Nonetheless, and also at mid-latitudes, there are cases of Es layers for which alternative formation processes are required: these include electric field-induced layers, HF and very high frequency (VHF) radar quasi-periodic echoing (QPE) regions, and ion rains (Mathews et al., 2001; Otsuka et al., 2007).

Concerning TILs specifically, there are still outstanding questions, for instance with respect to the confluence of the different tidal modes. With regard to this issue, Haldoupis et al. (2006) proposed a new methodology, the ionosonde height-time-intensity (HTI) analysis, for investigating the Es layer vertical motion and variability.

With this in mind, Es data recorded at mid-latitudes are considered in this study both to contribute additional information about the influence of tides on the Es layer, and to confirm that the HTI analysis represents a good alternative to study how the neutral wind dynamics affects the Es layer at mid-latitudes.

Section 2 describes the data set used, how the ionograms were validated, the HTI technique, and the fast Fourier transform (FFT) analysis. Section 3 has three main parts describing and discussing the results related to (1) the diurnal and monthly variability shown by the main characteristics of the Es layer, these are the top frequency (ftEs) and the lowest virtual height (h'Es); (2) the plots produced by the HTI analysis; and (3) the spectra produced by the FFT analysis. The conclusions form Sect. 4.

\section{Experiment, data, and analysis}

The analysis described in this paper is based on data recorded at two ionospheric stations: one in Rome (Italy, $41.8^{\circ} \mathrm{N}$, $12.5^{\circ} \mathrm{E}$ ) and another in Gibilmanna (Italy, $37.9^{\circ} \mathrm{N}, 14.0^{\circ} \mathrm{E}$ ), both equipped with an AIS-INGV (Advanced Ionospheric Sounder by Istituto Nazionale di Geofisica e Vulcanologia) ionosonde (Zuccheretti et al., 2003). A measurement campaign during the summer of 2013 (smoothed sunspot number $R_{12}=63$ ), from 4 June to the 30 September, was planned with a sounding repetition rate set to every $5 \mathrm{~min}$ at Rome and every $15 \mathrm{~min}$ at Gibilmanna, a sweeping frequency range from 1 to $16 \mathrm{MHz}$ at both stations, and with a frequency resolution of $0.05 \mathrm{MHz}$. The height range investigated by the AIS-INGV ionosonde was $90-760.5 \mathrm{~km}$ with a height resolution of $4.5 \mathrm{~km}$, these two values being fixed and depending on the architectural features of the ionosonde. The full frequency scale of the sounding was set to $16 \mathrm{MHz}$ because, as it is possible to infer from the visualization feature of the electronic Space Weather upper atmosphere database (eSWua; http://www.eswua.ingv.it/) (Romano et al., 2008), at Rome and Gibilmanna the Es layer has never shown critical frequency values of the Es ordinary mode (foEs) of propagation greater than $15 \mathrm{MHz}$.
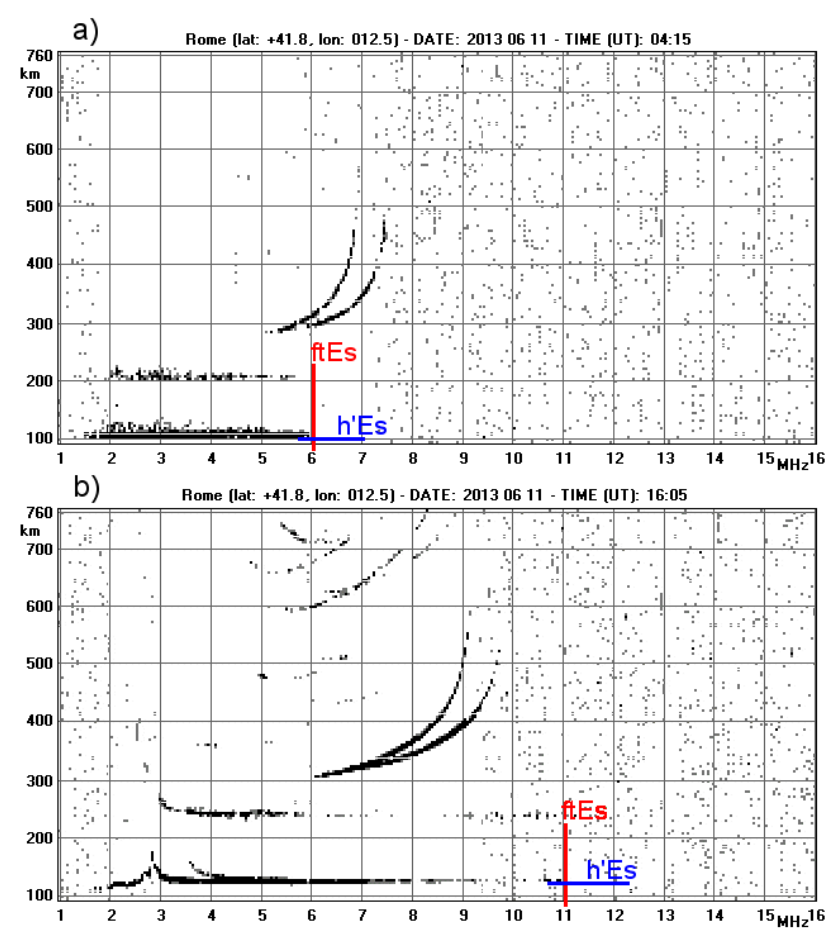

Figure 1. Examples of Es layer of (a) type "l" and (b) type "c" recorded at Rome in June 2013. The corresponding manually validated ftEs and h'Es are respectively shown in red and blue.

The summertime campaign was suggested by the wellknown fact that Es layer occurrence presents a maximum between May and September in the Northern Hemisphere, and between November and March in the Southern Hemisphere (e.g., Chu et al., 2014).

\subsection{Ionogram manual validation}

From each of the ionograms recorded during the whole campaign of 119 days at both stations, the ftEs and the h'Es characteristics were manually validated using the Interpre software developed by Pezzopane (2004). It was decided to refer to $\mathrm{ftEs}$ and not to foEs, because the AIS-INGV ionosonde cannot tag the different modes of propagation, that is the extraordinary and the ordinary ones, and for most of the ionograms it was practically impossible to distinguish between foEs and the critical frequency of the Es extraordinary mode (fxEs). The most frequent Es layers characterizing the ionograms recorded during the measurement campaign were of the "l" and the "c" types (Wakai et al., 1987), as shown in Fig. 1.

\subsection{Ionogram height-time-intensity (HTI) analysis}

In order to study how the atmospheric tides affect the Es dynamics, custom software was coded to perform the HTI analysis of the ionograms proposed by Haldoupis et al. (2006). 

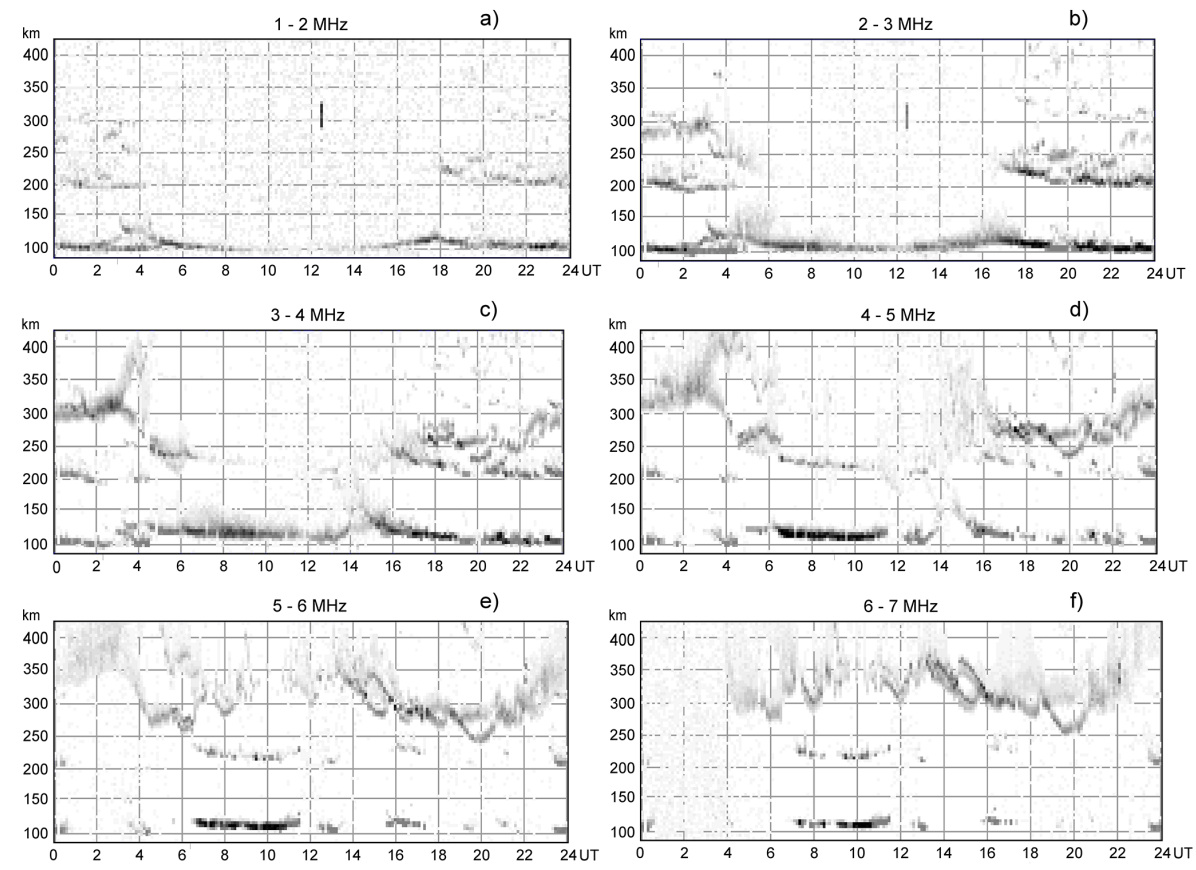

Figure 2. HTI plots averaged over two consecutive days, the 11 and 12 June 2013. Each plot corresponds to a different frequency range: (a) 1-2 MHz, (b) 2-3 MHz, (c) 3-4 MHz, (d) 4-5 MHz, (e) 5-6 MHz, and (f) 6-7 MHz.

An ionogram represents a snapshot of the ionosphere at the time of the sounding for a definite height and frequency range, hence from a single ionogram it would not be possible to derive the dynamics of the ionospheric layers. On the contrary, the HTI analysis considers a specific frequency range and, by using a series of ionograms, allows the study of the aforementioned dynamics. The output of the HTI analysis is a plot of the intensity of the energy received by the ionosonde, after being reflected by the ionosphere, versus the $24 \mathrm{~h}$ of the day, for a definite frequency range, and for the same height range of the ionogram.

Figure 2 shows HTI plots for different $1 \mathrm{MHz}$ frequency intervals, calculated by considering the ionograms recorded at Rome on the 11 and 12 June 2013. This means that each of the plots is based on the 576 ionograms recorded at Rome during the two considered days. The maximum of the height range, compared to that of the ionogram, is restricted to $400 \mathrm{~km}$ because the interest is in highlighting phenomena associated with Es layers that are characteristic of altitudes lower than $150 \mathrm{~km}$. Figure 2 shows that concerning the Es trace visible at altitudes between 100 and $150 \mathrm{~km}$, there are significant differences depending on the chosen frequency range. The HTI plots related to the frequency ranges 1-2 and $2-3 \mathrm{MHz}$ (Fig. 2a-b) are characterized by traces showing two maxima, approximately at 03:00 universal time (UT) (local time $(\mathrm{LT})=\mathrm{UT}+2)$ and 17:00-18:00 UT, while in the following plots the corresponding ascending traces disappear. As a first step, this phenomenon could be attributed to the Es layer, but a careful check of the ionograms reveals that this effect is actually caused by the cusp associated with the electron density maximum of the $\mathrm{E}$ region, which is characteristic of the Es layer of type "c" (see Fig. 1b). According to this consideration, the HTI analysis described in Sect. 3.2 refers mainly to frequency ranges higher than $3 \mathrm{MHz}$. Figure $2 \mathrm{c}-\mathrm{d}$ show instead two distinct Es layers that, starting from an altitude of about 125 and $150 \mathrm{~km}$ respectively at approximately 04:00 UT and 15:00 UT, lower to an altitude of about $100 \mathrm{~km}$ where they stop their descent. These descending traces are associated with the descent of the convergence node that characterizes the neutral wind profile connected with the atmospheric tides. This continues until the unloading altitude is reached, where the descent stops and the Es layer can remain for many hours (Chimonas and Axford, 1968).

Hence, as already shown by Haldoupis et al. (2006), the HTI plots are very useful when investigating Es layer dynamic features concurrently with the wind-shear theory.

Figure 2e-f show that, for higher frequency ranges, only the trace referring to the central hours of the day is visible. This is definitely due to the fact that the Es layer is much stronger during the day and weaker at night, because there are more metallic ions available during the day than at night from which the layer can form and intensify (Haldoupis et al., 2007). Additionally, this can likely be attributed to the confluence of the daytime semidiurnal trace with the diurnal trace that gives rise to the strongest Es layers observed during the day (Haldoupis et al., 2006). 

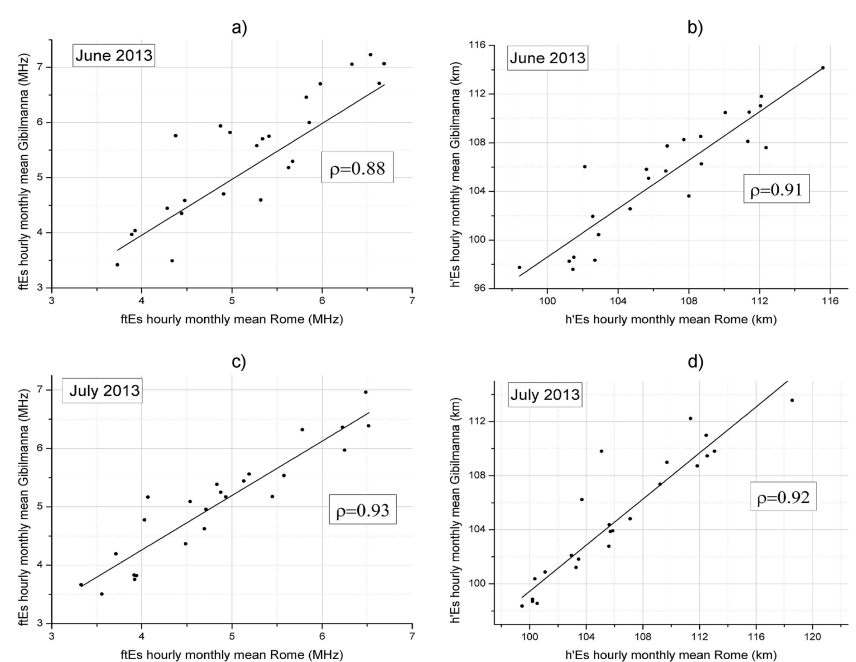

e)
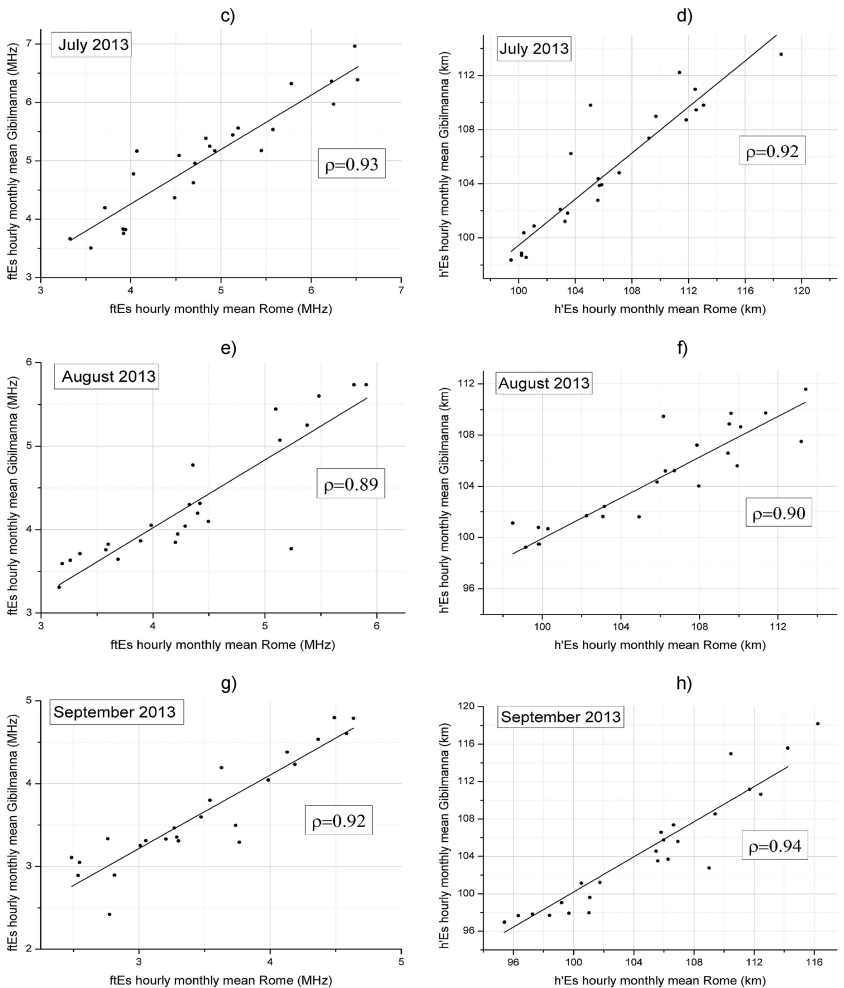

Figure 3. Linear correlation plots of the hourly monthly median $\mathrm{ftEs}$ and h'Es values recorded at Rome and Gibilmanna in (a-b) June, (c-d) July, (e-f) August, and (g-h) September.

In order to verify how the measurements recorded at Rome and Gibilmanna are correlated, the corresponding linear correlation plot of the hourly monthly median values of $\mathrm{ftEs}$ and h'Es was generated for each of the considered months, thus obtaining the corresponding linear correlation coefficient $\rho$ defined as

$\rho_{X, Y}=\frac{\operatorname{cov}(X, Y)}{\sigma_{X} \sigma_{Y}}=\frac{E((X-E(X))(Y-E(Y)))}{\sigma_{X} \sigma_{Y}}$,

where $\operatorname{cov}()$ is the covariance between the variables $X$ and $Y, \sigma_{X}$ and $\sigma_{Y}$ are the corresponding standard deviations, and $E()$ represents the expected value. Figure 3 shows that $\rho$ ranges from 0.88 to 0.94 , which means that the two data sets of measurements are highly correlated. In light of this, and also taking into account that the sounding repetition rate of the AIS-INGV ionosonde installed at Rome is higher ( $5 \mathrm{~min})$ and consequently better resolves the Es dynamics, the HTI analysis described in the Sect. 3.2 focuses on the ionograms recorded at Rome.

\subsection{The fast Fourier transform analysis}

The FFT analysis decomposes the time-domain signal into complex exponentials. A Fourier transform offers a complete picture of the frequency space, but it does not give any information about the time evolution of the signal. This means that the FFT analysis is mostly used to investigate stationary signals.

Specifically, the FFT is an optimized algorithm for calculating the discrete Fourier transform and its inverse, which are defined as follows:

$X_{k}=\sum_{n=0}^{N-1} x_{n} e^{\frac{i 2 \pi k n}{N}}$

where $x_{0}, x_{1}, \ldots, x_{N-1}$ is a sequence of complex numbers and $k=0, \ldots, N-1$;

$x_{n}=\frac{1}{N} \sum_{k=0}^{N-1} X_{k} e^{-\frac{i 2 \pi k n}{N}}$,

with $n=0, \ldots, N-1$.

In order to study the main periodicities imprinted in the Es layer data, in Sect. 3.3 the FFT analysis is used to compute the amplitude spectra of the hourly mean values of ftEs and h'Es for each considered month.

The FFT method proved to be suitable for this study because the tidal waves continuously affect the Es layers with diurnal and semidiurnal periodicities (Haldoupis et al., 2004, 2006), and then the phenomenon can be considered as stationary. In order to reduce data gaps caused by the absence of the Es layer, the ftEs and h'Es hourly mean values were considered. In this way, only a few data gaps were left, mostly in late August and September, and in order to work with a complete time series they were filled with the corresponding monthly mean values. Moreover, to avoid edge problems and cut problems that can affect the FFT method, the corresponding mean value was subtracted from the $\mathrm{ftEs}$ and the h'Es hourly mean time series of each month, obtaining as a consequence two zero mean time series, and zeros at the beginning and at the end of the time series were added up to reach the nearest multiple of 2 (1024 in our case).

\section{Results and discussion}

\subsection{The Es diurnal and monthly variability}

Figures 4 and 5 show respectively the ftEs and h'Es hourly monthly mean values with the corresponding standard deviation, the occurrence frequency of the Es layer, and the relative frequency of the ftEs and h'Es hourly mean values for June, July, August and September 2013, for Rome (in 
a)

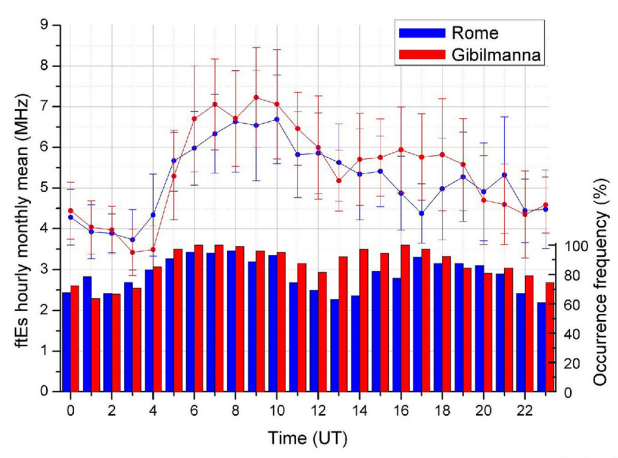

c)

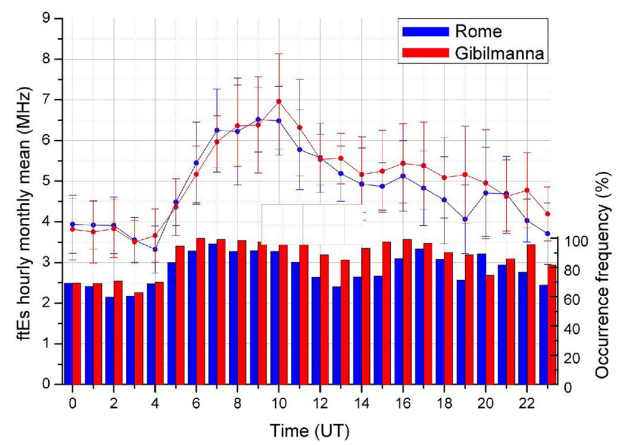

e)

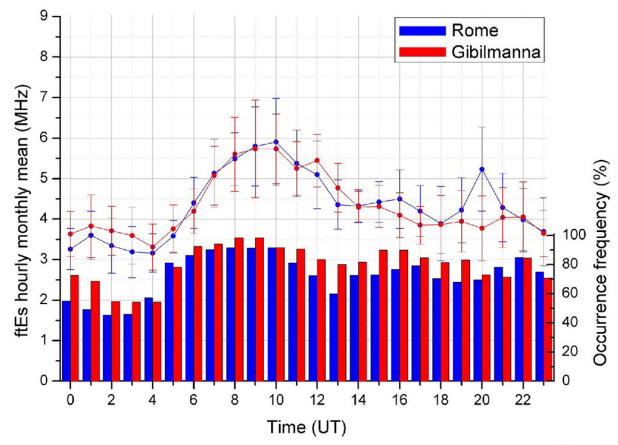

g)

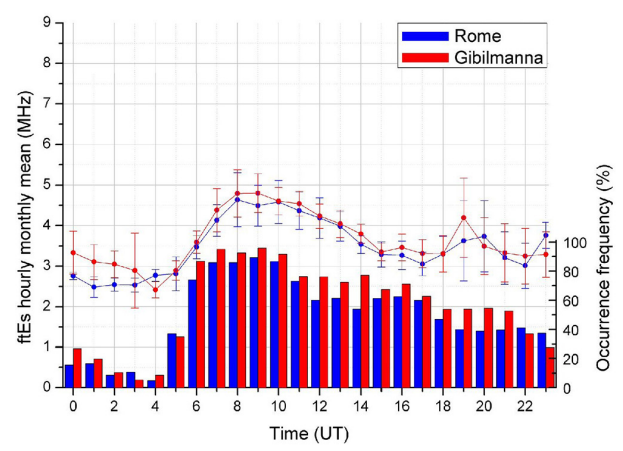

June 2013

July 2013

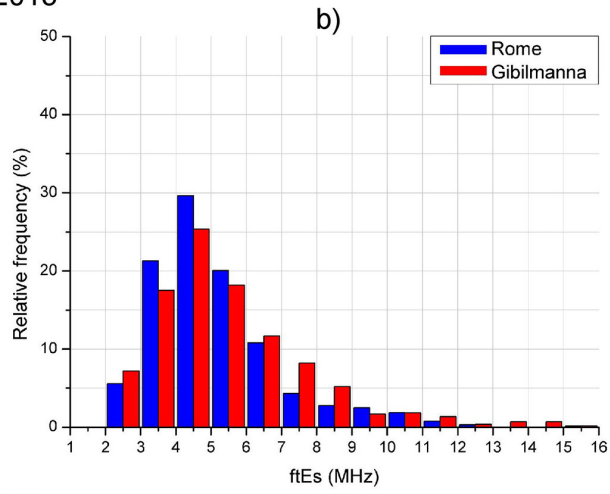

d)

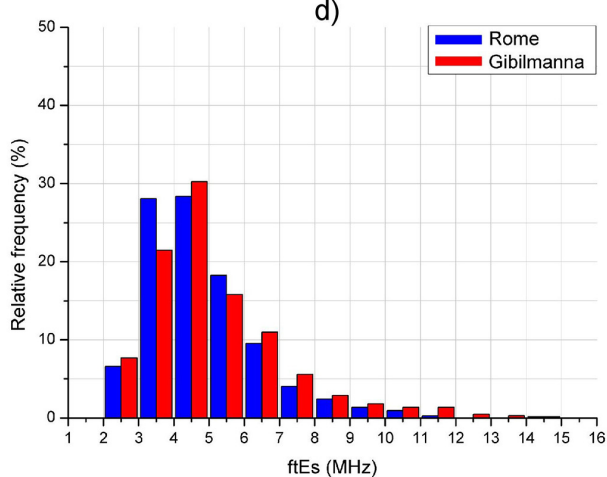

August 2013

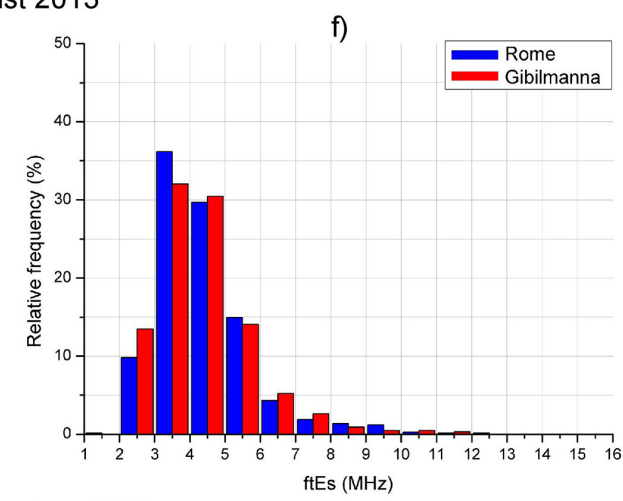

September 2013



Figure 4. ftEs hourly monthly mean values with the corresponding standard deviation along with the occurrence frequency of the Es layer for (a) June, (c) July, (e) August and (g) September 2013, and the relative frequency of the ftEs hourly mean values for (b) June, (d) July, (f) August and (h) September 2013, for Rome (in blue) and Gibilmanna (in red). 
blue) and Gibilmanna (in red). For each interval of $1 \mathrm{MHz}$, the relative frequency was calculated as the ratio between the hourly mean values ranging from $f .00 \mathrm{MHz}$ to $f .99 \mathrm{MHz}$ (with $f=1, \ldots, 15$ ) and the total number of hourly mean values.

In June ftEs (Fig. 4a) presents a daily trend characterized by a large maximum around 10:00 UT for both stations, and a much smaller maximum at 18:00-21:00 UT for Rome and at 15:00-19:00 UT for Gibilmanna, while h'Es (Fig. 5a) presents a daily trend characterized by two similar maxima at 05:00 and 15:00-16:00 UT for both sites. The occurrence frequency shows a trend characterized by two maxima with values ranging from 60 to $100 \%$. In particular, the occurrence frequency recorded at Rome is lower than that recorded at Gibilmanna, confirming the recent findings of Pietrella et al. (2014). The relative frequency of the hourly mean values in June presents a similar trend at both sites and has a maximum for ftEs between 4 and $5 \mathrm{MHz}$ (Fig. 4b) and for h'Es between 100 and $105 \mathrm{~km}$ (Fig. 5b).

In July (Figs. $4 \mathrm{c}-\mathrm{d}$ and $5 \mathrm{c}-\mathrm{d}$ ) all the trends are by and large very similar to those recorded in June, with the only exception of the ftEs relative frequencies that are slightly shifted to lower values.

In August the trends continue to be similar, but the values of ftEs are lower than those recorded in June and July. The maximum of $\mathrm{ftEs}$ visible at 10:00 UT is now equal to about $6 \mathrm{MHz}$ (Fig. 4e), and the corresponding relative frequency presents a maximum between 3 and $4 \mathrm{MHz}$ (Fig. 4f). On the contrary, h'Es does not exhibit any appreciable difference; in fact, in spite of a decrease in the Es layer occurrence frequency, the corresponding semidiurnal trend is always well defined with both the position and the value of the two maxima similar to those of the previous months (Fig. 5e-f).

In September, even though the semidiurnal trend is still clearly visible, the decrease of the ftEs values continues, more pronouncedly during the daytime, showing a maximum value lower than $5 \mathrm{MHz}$ (Fig. $4 \mathrm{~g}$ ), and a corresponding maximum of the relative frequency between 3 and $4 \mathrm{MHz}$ (Fig. 4h), like in August but more pronounced. In this month, the Es occurrence frequency also decreases significantly, almost disappearing in the nighttime. The h'Es trend shows a daily trend similar to those of the previous months, while both a decrease in the Es occurrence frequency and a slight shifting of the relative frequency to lower values are observable.

\subsection{The influence of tides on Es dynamics: the HTI plots}

Figures $6,7,8$, and 9 show four examples of the HTI analysis, one for each of the considered months, and representative of the modality by which the Es dynamics manifests for most of the days of that month. Specifically, for each day analyzed the following outcomes are shown: (1) the HTI plots from which, besides studying the Es layer dynamics, it is possible to deduce how the Es layer intensity changes for different frequency ranges; (2) the daily trend of the h'Es values that were manually validated from the ionograms, in order to prove what is perceivable by the HTI plots. The aim of this analysis is to investigate the efficiency of the windshear mechanism as the dynamic conditions of the neutral atmosphere change.

Figure 6a depicts the h'Es trend obtained after manually analyzing all the ionograms recorded on the 6 June 2013 at Rome, and shows three distinct descending traces for which the linear fits were calculated. This analysis was performed with an awareness that the Es layer forms in the convergence node of the neutral wind profile associated with tides (Whitehead, 1961; Axford, 1963) and then, between 100 and $130 \mathrm{~km}$, it is dragged to lower altitudes till the unloading altitude is reached at about $100 \mathrm{~km}$, where the descent stops and the Es layer stands for many hours (Chimonas and Axford, 1968). Hence, the Es layer descent patterns are approximately represented by the linear fits represented in Fig. 6a, the negative angular coefficient of which represents the Es descent velocity that, according to the wind-shear theory, is equivalent to the phase velocity of the convergence node characterizing the neutral wind profile associated with the tides (Christakis et al., 2009). The first trace appears at about 04:00 UT, at an altitude of $126 \mathrm{~km}$, and descends with a medium velocity of $(-6.4 \pm 0.5) \mathrm{km} \mathrm{h}^{-1}$ to an altitude of $105 \mathrm{~km}$ at 08:00 UT; the second trace appears at about 10:00 UT, at an altitude of $110 \mathrm{~km}$, and descends with a medium velocity of $(-1.3 \pm 0.1) \mathrm{km} \mathrm{h}^{-1}$ to an altitude of $100 \mathrm{~km}$ at 17:00 UT; the third trace appears at about 18:00 UT, at an altitude of $120 \mathrm{~km}$, and descends with a medium velocity of $(-3.6 \pm 0.4) \mathrm{km} \mathrm{h}^{-1}$ to an altitude of $103 \mathrm{~km}$ at 21:00 UT. The first and the third traces are separated in time by $12 \mathrm{~h}$ and, by also taking into account the velocity characterizing the descent, this is a signal that they are strongly connected to the semidiurnal tides (Forbes, 1995); the corresponding estimated wavelengths are respectively $(77 \pm 7) \mathrm{km}$ and $(44 \pm 6) \mathrm{km}$. The second trace is associated with the diurnal tide, characterized by a velocity lower than the semidiurnal tide, and the estimated wavelength is in this case equal to $(33 \pm 4) \mathrm{km}$. The diurnal trace can be associated with the diurnal tide mode $S(1,1)$, while the daytime and nighttime semidiurnal traces can be associated respectively to the semidiurnal tide modes $\mathrm{S}(2,3)$ and $\mathrm{S}(2,5)$ (Holton, 1975; Forbes, 1995). Figure 6b-e show the HTI plots related to the 6 June 2013 from which a well defined semidiurnal pattern appears for low frequency ranges at altitudes typical of the $\mathrm{E}$ region; this pattern then disappears for higher frequency ranges, when only a trace between 08:00 and 12:00 UT is visible, likely associated with the fusion of the daytime semidiurnal and diurnal traces that contributes to the $\mathrm{ftEs}$ maximum at about 10:00 UT found for all the investigated months (see Fig. 4a, c, e, f). No clear diurnal pattern was made clear by the HTI analysis and this can be ascribed, on the one hand, to the fact that the corresponding layer acts at lower heights and at times may become too weak to be 


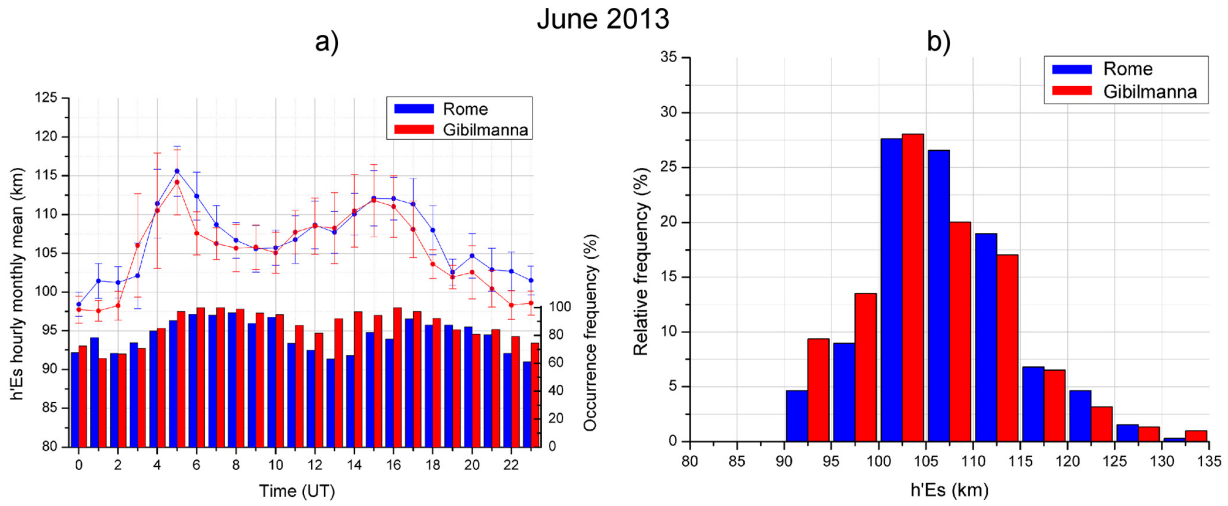

c)

July 2013
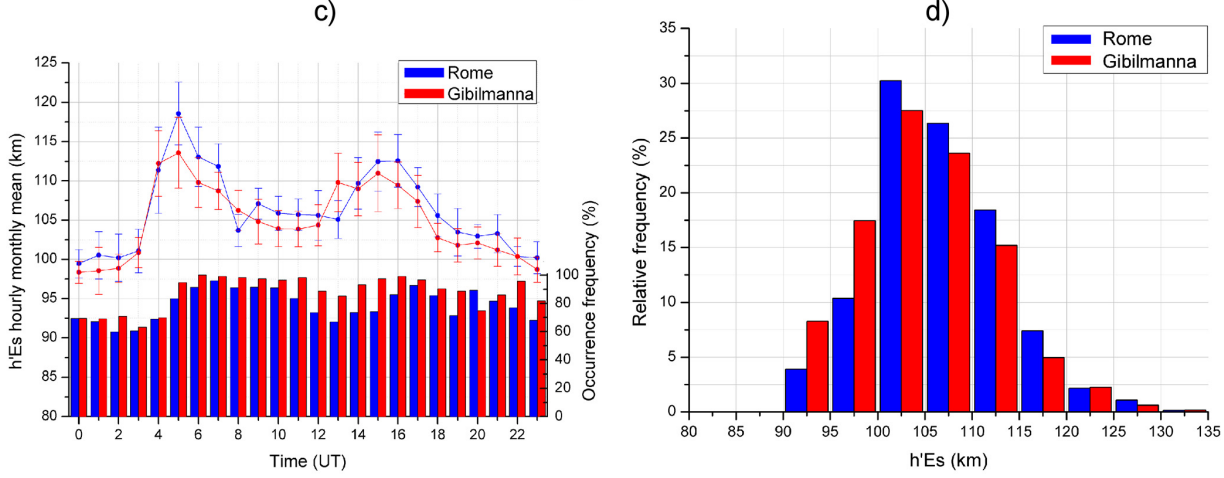

e)

August 2013
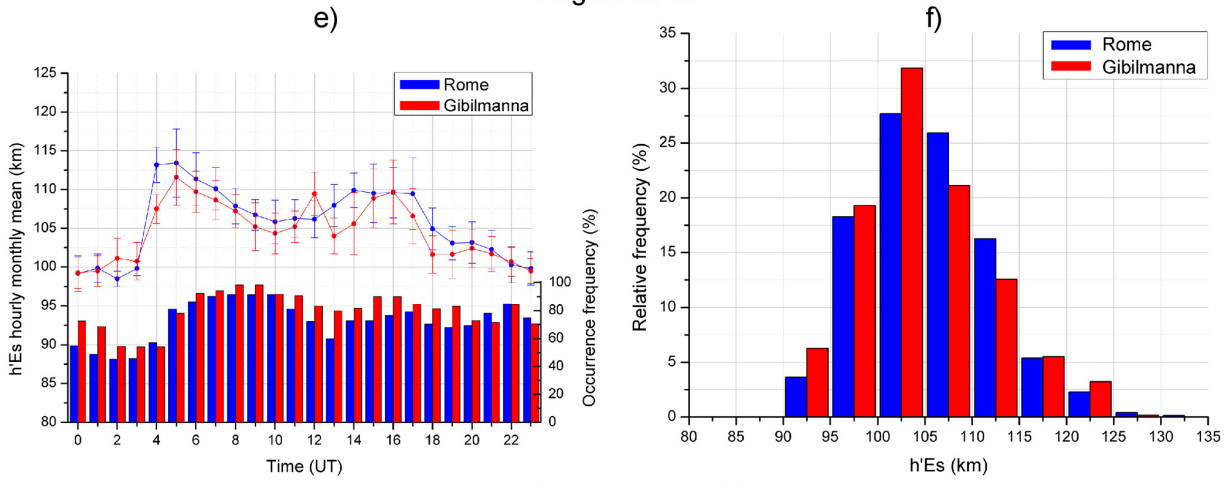

g)

September 2013
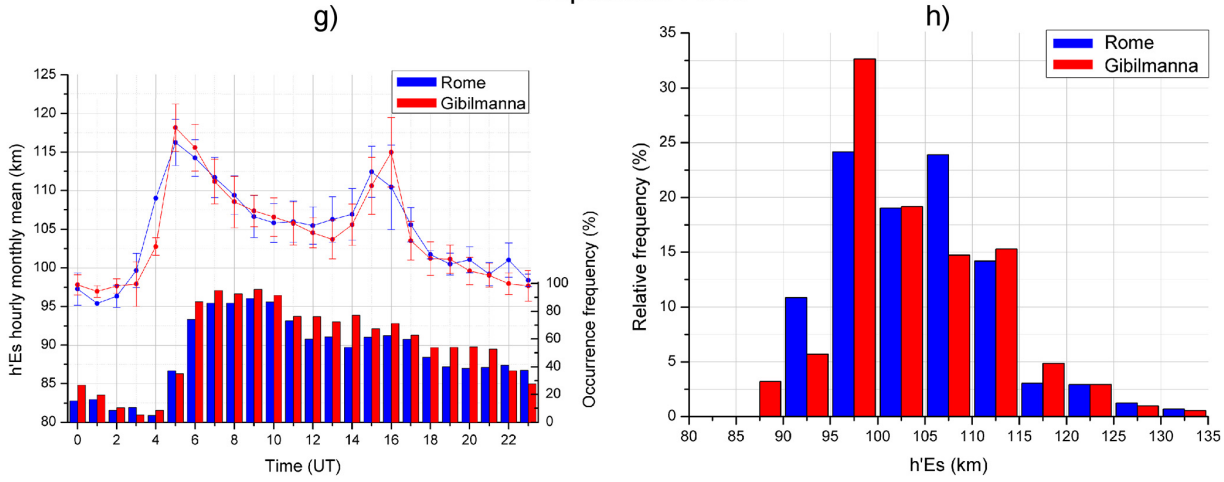

Figure 5. h'Es hourly monthly mean values with the corresponding standard deviation along with the occurrence frequency of the Es layer for (a) June, (c) July, (e) August and (g) September 2013, and the relative frequency of the h'Es hourly mean values for (b) June, (d) July, (f) August and (h) September 2013, for Rome (in blue) and Gibilmanna (in red). 
a)



b)



d)

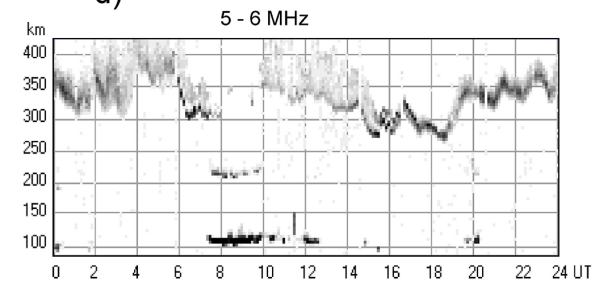

c)

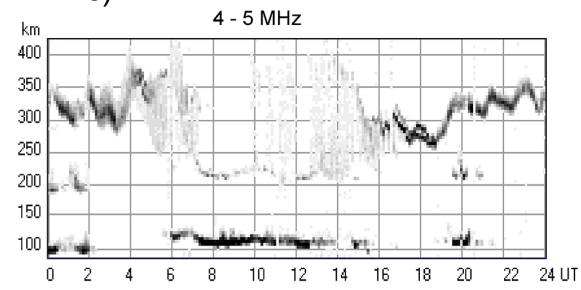

e)

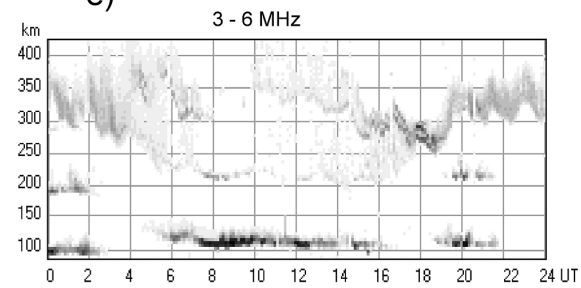

Figure 6. (a) h'Es values manually validated for the 6 June 2013, with the corresponding linear fits, and the HTI plots generated for the same day for different frequency ranges: (b) 3-4 MHz, (c) 4-5 MHz, (d) 5-6 MHz, and (e) 3-6 MHz.

detected by the ionosonde, and, on the other hand, to the fact that the height resolution of the AIS-INGV ionosonde is limited to $4.5 \mathrm{~km}$. (A height resolution of $1.5 / 2.0 \mathrm{~km}$ would be more appropriate.)

Figure 7a depicts the h'Es trend obtained after manually analyzing all the ionograms recorded the 2 July 2013 at Rome, and shows two distinct descending traces for which linear fits were calculated. The first trace appears at about 04:00-05:00 UT, at an altitude of $130 \mathrm{~km}$, and it descends with a medium velocity of $(-5.3 \pm 0.5) \mathrm{km} \mathrm{h}^{-1}$ to an altitude of $102 \mathrm{~km}$ at 10:00 UT; the second trace appears at about 14:00 UT, at an altitude of $120 \mathrm{~km}$, and it descends with a medium velocity of $(-2.5 \pm 0.2) \mathrm{km} \mathrm{h}^{-1}$ to an altitude of $99 \mathrm{~km}$ at 21:00 UT. The corresponding estimated wavelengths are respectively $(64 \pm 7) \mathrm{km}$ and $(30 \pm 3) \mathrm{km}$. The pattern in this case is clearly semidiurnal; the daytime semidiurnal trace can be associated with a tide mode between $S(2,3)$ and $S(2,4)$, while the nighttime semidiurnal trace can be associated with the tide mode $\mathrm{S}(2,6)$ (Holton, 1975; Forbes, 1995). Figure 7b-e show the HTI plots related to the 2 July 2013, which confirm the well defined semidiurnal pattern characterizing the $\mathrm{E}$ region altitudes mostly for low frequency ranges.

Figure 8a, depicting the h'Es trend obtained after manually analyzing all the ionograms recorded the $21 \mathrm{Au}-$ gust 2013 at Rome, shows two distinct descending traces for which linear fits were calculated. The first trace appears at about 04:00 UT, at an altitude of $112 \mathrm{~km}$, it descends with a medium velocity of $(-0.9 \pm 0.1) \mathrm{km} \mathrm{h}^{-1}$ to an altitude of $102 \mathrm{~km}$ at 14:00 UT, and can be associated with the diurnal trace, apparent from the values of the medium velocity and the starting altitude of descent; the second trace appears at about 15:00 UT, at an altitude of $117 \mathrm{~km}$, it descends with a medium velocity of $(-2.4 \pm 0.2) \mathrm{km} \mathrm{h}^{-1}$ to an altitude of $100 \mathrm{~km}$ at 21:00 UT, and can be associated with the nighttime semidiurnal trace. The corresponding estimated wavelengths are respectively $(24 \pm 4) \mathrm{km}$ and $(29 \pm 3) \mathrm{km}$. Hence, with respect to the previous two cases, the daytime semidiurnal trace is absent, with a consequent reinforcement of the diurnal trace. The diurnal trace can be associated with the tide mode $\mathrm{S}(1,1)$, while the nighttime semidiurnal trace can be associated with the tide mode $\mathrm{S}(2,6)$ (Holton, 1975; Forbes, 
a)

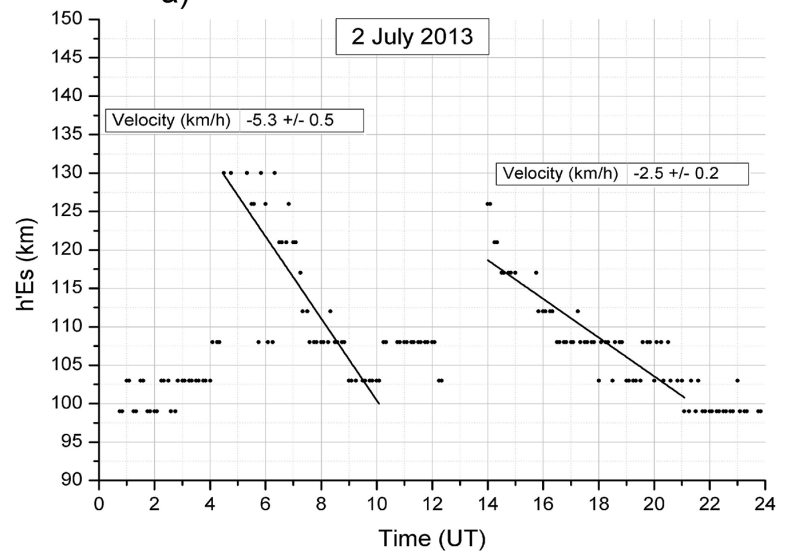

b)

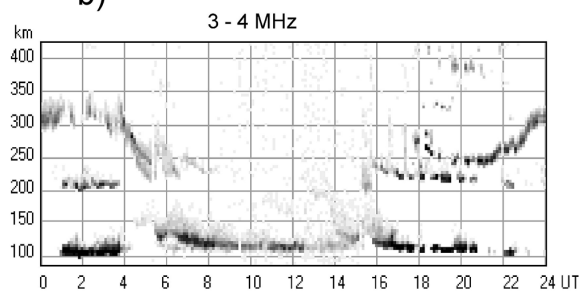

d)

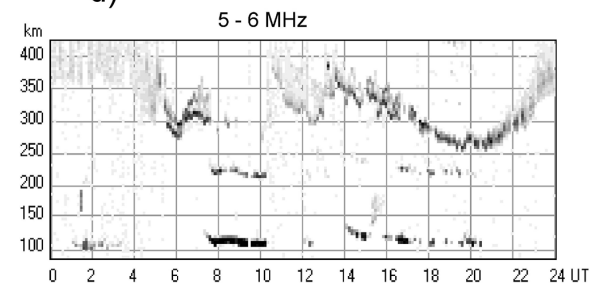

c)

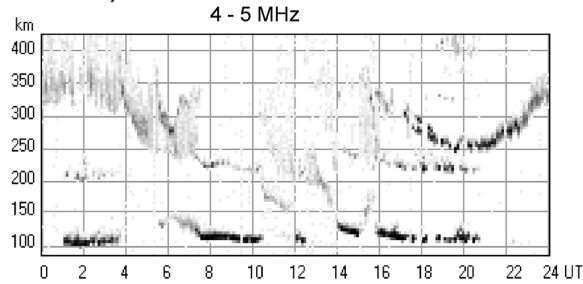

e)

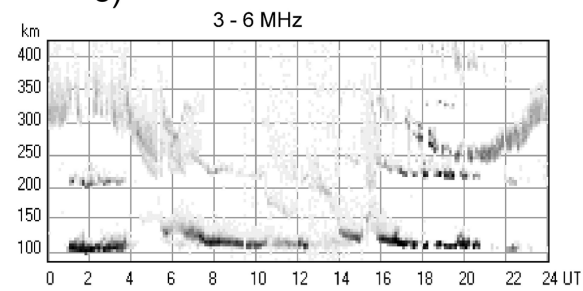

Figure 7. (a) h'Es values manually validated for the 2 July 2013, with the corresponding linear fits, and the HTI plots generated for the same day for different frequency ranges: (b) 3-4 MHz, (c) 4-5 MHz, (d) 5-6 MHz, and (e) 3-6 MHz.

1995). The lack of the daytime semidiurnal trace is also proved by the HTI plots shown in Fig. 8b-e from which at altitudes typical of the E region, unlike the HTI plots shown in Figs. 6 and 7, the trace associated with the descent of the daytime semidiurnal tide is no longer visible. One could think that the daytime semidiurnal trace is screened by an unusually intense diurnal trace; however, this hypothesis can be discarded because the HTI plots of Fig. $8 \mathrm{c}-\mathrm{d}$ show that between 09:00 an 14:00 UT, where the main maximum of ftEs is usually visible for higher frequency ranges, no maximum is present. On the other hand, this feature can be attributed to the absence of the daytime semidiurnal tide which, not giving its ionic contribution in conjunction with the diurnal trace, causes lower values of ftEs than are usually found. On the contrary, the nighttime semidiurnal trace, as was previously found in Figs. 6 and 7, is so intense that it dominates the HTI plots of Fig. 8c-d.

Figure 9a, depicting the h'Es trend obtained after manually analyzing all the ionograms recorded on the 3 September 2013 at Rome, shows that the reinforcement of the diurnal trace continues: the semidiurnal traces are in this case both absent. The diurnal trace appears at about 06:00 UT, at an altitude of $112 \mathrm{~km}$, it descends with a medium velocity of $(-1.7 \pm 0.1) \mathrm{km} \mathrm{h}^{-1}$ to an altitude of $95 \mathrm{~km}$ at 18:00 UT, and it can be associated with the tide mode $\mathrm{S}(1,1)$ (Holton, 1975; Forbes, 1995). The corresponding estimated wavelength is $(42 \pm 3) \mathrm{km}$. It is worth noting that the unloading altitude is in this case lower than those characterizing the previous cases, and this is correct due to the missing action performed by the semidiurnal tides, which are characterized by an unloading altitude higher than that of the diurnal tide (Haldoupis et al., 2006; Christakis et al., 2009). In fact, the semidiurnal tides influence the Es layer dynamics at altitudes higher than $110 \mathrm{~km}$, while the diurnal tide influences the Es layer dynamics at altitude lower than $110 \mathrm{~km}$ and, in the absence of other dynamical inputs, can drag the Es layer down to altitudes lower than $90 \mathrm{~km}$. The predominance of the diurnal trace is also highlighted by the HTI plots shown in Fig. 9b-e where neither semidiurnal traces is visible. 
a)



b)

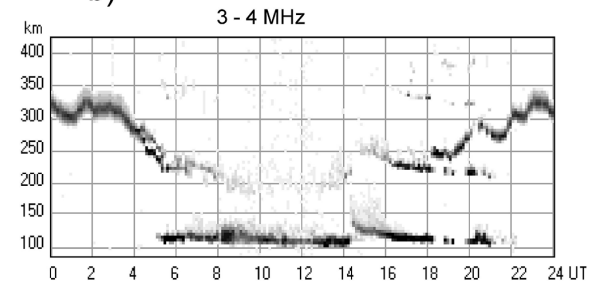

d)

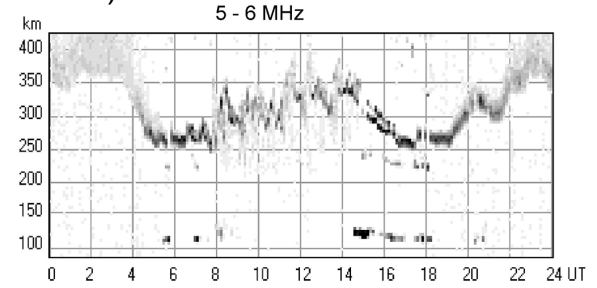

c)

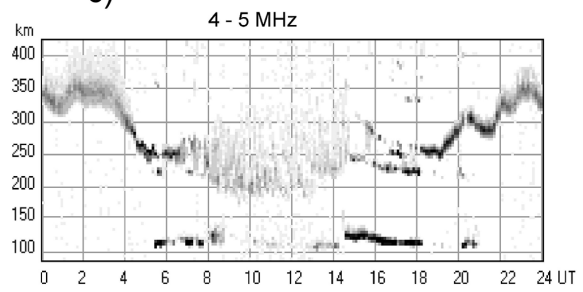

e)

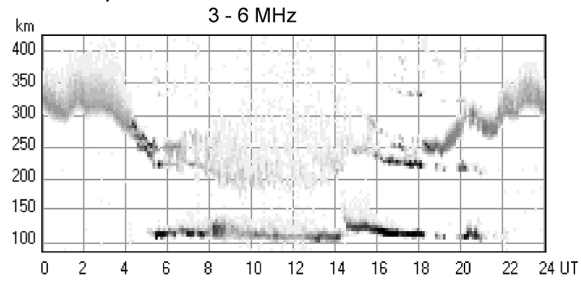

Figure 8. (a) h'Es values manually validated for the 21 August 2013, with the corresponding linear fits, and the HTI plots generated for the same day for different frequency ranges: (b) 3-4 MHz, (c) 4-5 MHz, (d) 5-6 MHz, and (e) 3-6 MHz.

\subsection{The tidal periodicities imprinted in the Es data: the fast Fourier transform analysis}

The HTI analysis described in Sect. 3.2 demonstrated that the influence of the atmospheric tides presents a seasonal dependence: in June and July the influence of semidiurnal tides is prevalent, in August from time to time the daytime semidiurnal tide is absent and consequently the diurnal tide becomes more evident, and in September the diurnal tide tends to prevail. With regard to this, and in order to have a vision as comprehensive as possible, Fig. 10 shows the FFTs of the ftEs and h'Es hourly mean values recorded for each month at Rome. It is worth noting that the h'Es FFT spectra of Fig. 10b, d, f, h, besides being characterized by well defined diurnal and semidiurnal periodicities, also highlight terdiurnal ( $8 \mathrm{~h}$ period) and quarter-diurnal $(6 \mathrm{~h}$ period) periodicities. The HTI analysis shown in Sect. 3.2, unlike the work of Haldoupis et al. (2006), did not succeed in highlighting these periodicities.
Figure 10a-b show respectively the ftEs and h'Es FFT spectra for June 2013 for the periods between 2 and $36 \mathrm{~h}$ (the dashed line indicates the $95 \%$ level of confidence). Figure 10a shows two maxima at 12 and $24 \mathrm{~h}$, with the latter being the largest and corresponding to the main maximum visible in Fig. 4a at around 10:00 UT. The fact that the maximum at $24 \mathrm{~h}$ is more intense and extended than that at $12 \mathrm{~h}$ is certainly due to the fact that there are more metallic ions available during the day than at night, from which the layer can form and intensify (Haldoupis et al., 2007), but also because both the diurnal Es layer and the daytime semidiurnal Es layer contribute to it. Figure 10b shows that for h'Es the two maxima at 12 and $24 \mathrm{~h}$ are comparable, a fact that was also highlighted by Fig. 6, where the corresponding analysis showed the presence of semidiurnal traces along with a weak diurnal trace that is often screened by the daytime semidiurnal trace. The difference in intensity between $\mathrm{ftEs}$ and h'Es is due to the fact that h'Es is exclusively a spatial characteristic directly connected to the Es dynamics, and hence directly affected by the semidiurnal and diurnal tides, 
a)



b)

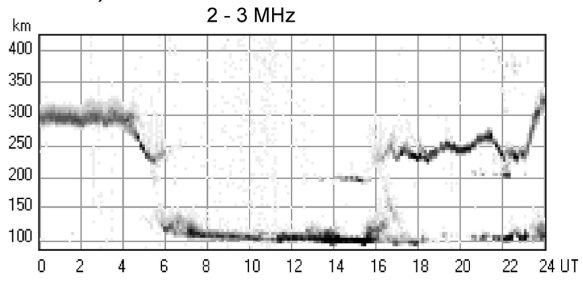

d)

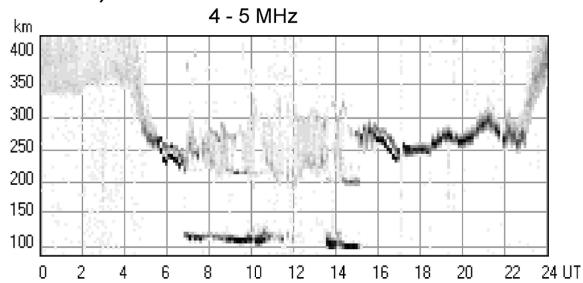

c)

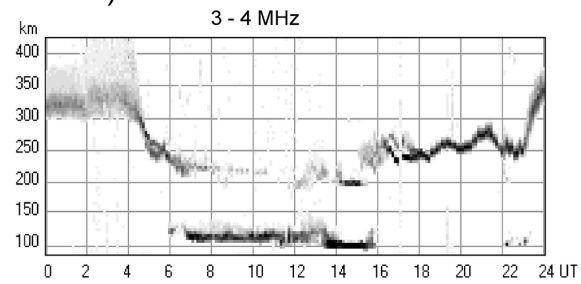

e)



Figure 9. (a) h'Es values manually validated for the 3 September 2013, with the corresponding linear fits, and the HTI plots generated for the same day for different frequency ranges: (b) 2-3 MHz, (c) 3-4 MHz, (d) 4-5 MHz, and (e) 2-5 MHz.

while ftEs, in addition to the Es dynamics, is mostly associated with the ionic content and hence to the accumulation of the ions caused by the wind-shear mechanism. As a consequence, the FFT periodicities associated with these two parameters, although they are mainly semidiurnal and diurnal and associated with tides, can present different intensities and extensions. Concerning June, it is worth remarking again that the diurnal periodicity of $\mathrm{ftEs}$ is correct, due to the intense occurrence of semidiurnal tides, as highlighted by the h'Es spectrum, and not to a predominant diurnal tide; in fact, as it should be clear by now, the main maximum of ftEs shown in Fig. 4a, besides being due to more metallic ions available during the day than at night, is also due to the confluence of the daytime semidiurnal and diurnal tides and not to the sole diurnal tide.

The same considerations made for June can also be made for July (Fig. 10c-d). The h'Es spectrum (Fig. 10d), showing a $12 \mathrm{~h}$ maximum larger than that at $24 \mathrm{~h}$, confirms the findings of the analysis shown in Fig. 7 where the prevalence of the semidiurnal traces is well defined. Hence, as for June, the definite diurnal periodicity of the ftEs spectrum, which gives rise to the maximum shown in Fig. 4c, besides being due to the usual daily cycle, can also be attributed to a mixing of the diurnal and the semidiurnal layers.

Figure 10e-f show the ftEs and h'Es FFT spectra for August 2013, always for the periods between 2 and $36 \mathrm{~h}$. In this case the ftEs spectrum presents two maxima at 12 and $24 \mathrm{~h}$ that are almost the same, meaning that the daytime semidiurnal trace became weaker, which is also perceivable from the HTI analysis shown in Fig. 8. This feature was also apparent from the h'Es spectrum shown in Fig. 10f where, unlike the previous months, the periodicity at $24 \mathrm{~h}$ is larger than that at $12 \mathrm{~h}$.

Figure $10 \mathrm{~g}-\mathrm{h}$ show the ftEs and h'Es FFT spectra for September 2013, again for the periods between 2 and $36 \mathrm{~h}$. The behavior seen in August is confirmed and strengthened in this month. In fact, like in August, the ftEs spectrum (Fig. $10 \mathrm{~g}$ ) presents two maxima at 12 and $24 \mathrm{~h}$ that are almost comparable, and the h'Es spectrum (Fig. 10h) presents 

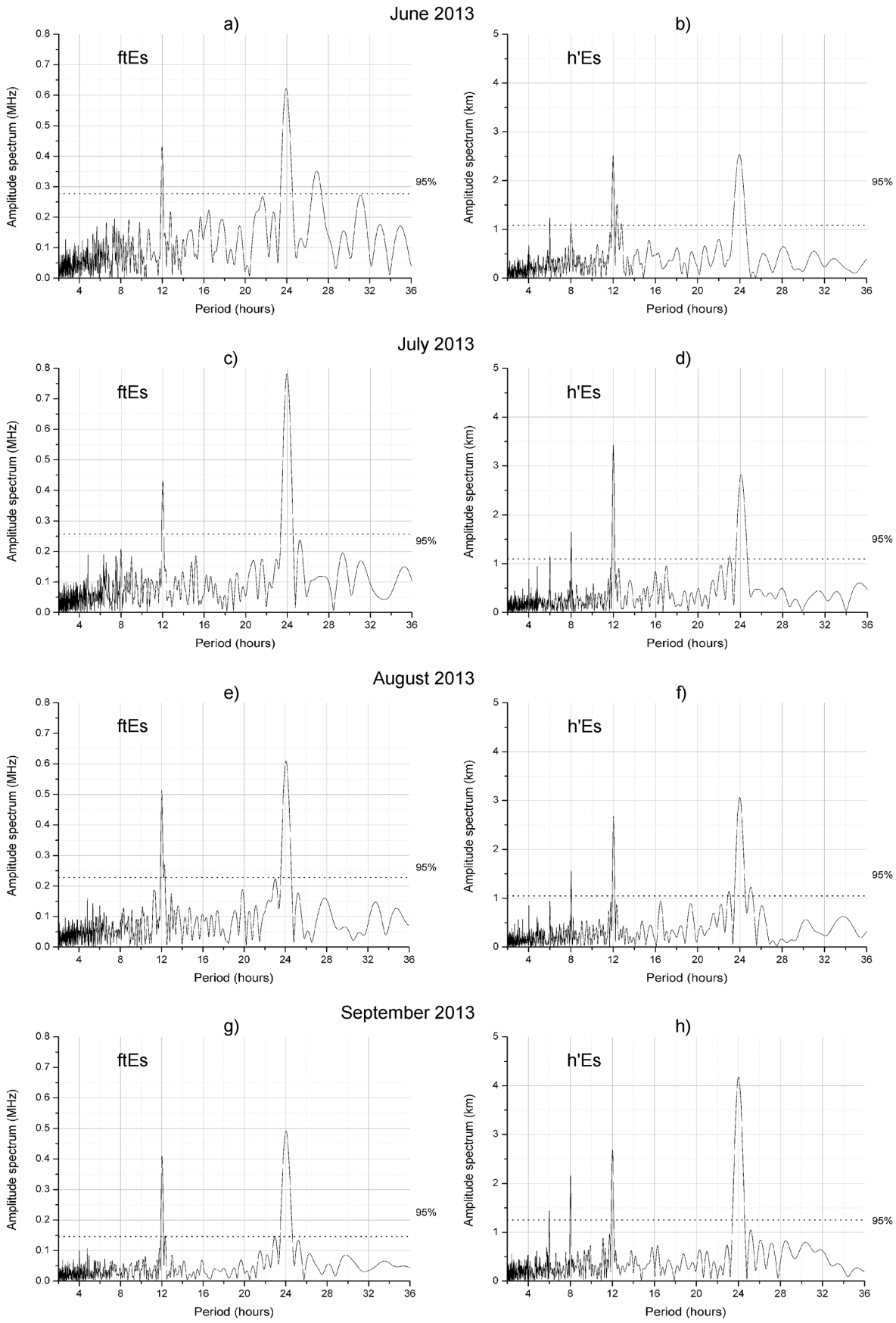

Figure 10. FFTs of the ftEs and h'Es hourly mean values recorded at Rome in (a-b) June, (c-d) July, (e-f) August, and (g-h) September 2013.

a well defined diurnal periodicity, as already shown in Fig. 9 by the HTI analysis.

\section{Conclusions}

Using ionograms recorded at Rome and Gibilmanna during the summertime of 2013, the average properties of the Es layer were studied and the HTI technique was used to investigate how tides affect the Es dynamics. The main results of the study are as follows. 
1. The ftEs characteristic, associated with the ionic content of the ionospheric $\mathrm{E}$ region, presents its maximum values in June and July, confirming this well known feature of the mid-latitude Es layer (e.g., Chu et al., 2014). The Es occurrence frequency also shows a behavior which is very similar to that of $\mathrm{ftEs}$, with variations that are significant mostly during nighttime hours for which the Es layer is almost absent in August and September. In daytime hours the Es occurrence frequency shows that the Es layer is a regularly occurring phenomenon also around the winter equinox, even with values of the critical frequency lower than those characterizing June and July. Haldoupis et al. (2007) have recently proposed the meteoric influx as a justification of this seasonal dependency of the Es.

2. Besides the main maximum shown at about 10:00 UT, ftEs also presents a secondary maximum characterized by a more pronounced variability. In fact, the position of this maximum is highly variable through the months. However, this should not be a surprise, because the presence of these maxima is caused by the efficiency of the wind-shear mechanism in accumulating ions in a thin ionospheric region, and this mechanism is affected by the descent of the convergence nodes of atmospheric tides. Hence, it could be inferred that the main maximum, mainly related to the greater availability of metallic ions during the day than at night, is also caused by the confluence of the diurnal and the daytime semidiurnal tides that therefore act simultaneously in accumulating ions. Meanwhile, the secondary maximum is caused only by the nighttime semidiurnal tide, which would justify the correspondingly more pronounced variability (Haldoupis et al., 2006).

3. Unlike ftEs, h'Es presents similar trends for all the considered months, with two comparable maxima that remain more or less unchanged going from June to September. This fact would be the manifestation of a certain stability of the mechanism affecting the Es dynamics, which is on the other hand strongly influenced by tides.

4. The HTI analysis (Haldoupis et al., 2006) showed that a well-defined semidiurnal periodicity characterizes the Es layer descent and occurrence mostly in June and July, while in August and September the daytime semidiurnal periodicity becomes weaker and the role of the diurnal periodicity is consequently highlighted.

5. The HTI method has proven itself to be a good tool for analyzing large data sets of ionograms in order to investigate the neutral wind dynamics at $\mathrm{E}$ region altitudes. In fact, unlike the manual validation of ionograms, an unquestionable quality of the HTI method lies in the rapidity by which the $\mathrm{E}$ region dynamics is highlighted.
It is worth noting however that the height resolution characterizing the AIS-INGV ionosonde, which is equal to $4.5 \mathrm{~km}$, certainly constrains the study of the Es dynamics, because the thickness of the Es layer is of the order of few kilometers, and most of Es movements occur within the AIS-INGV height resolution. The HTI method was shown in this case to be a powerful tool that can highlight the important features of the Es dynamics. Moreover, the HTI technique can be supplementary to the powerful Incoherent Scattering Radar (ISR) technique because, even though the information coming from an ISR measurement is certainly more comprehensive, an ionosonde has the advantage of making the long-term measurements which are necessary for studying tidal and planetary wave effects on ionospheric plasma.

Topical Editor S. Milan thanks two anonymous referees for their help in evaluating this paper.

\section{References}

Axford, W. I.: The formation and vertical movement of dense ionized layers in the ionosphere due to neutral wind shears, J. Geophys. Res., 68, 769-779, 1963.

Chimonas, G. and Axford, W. I.: Vertical movement of temperatezone sporadic E layers, J. Geophys. Res., 73, 111-117, 1968.

Christakis, N., Haldoupis, C., Zhou, Q., and Meek, C.: Seasonal variability and descent of mid-latitude sporadic E layers at Arecibo, Ann. Geophys., 27, 923-931, 2009, http://www.ann-geophys.net/27/923/2009/.

Chu, Y. H., Wang, C. Y., Wu, K. H., Chen, K. T., Tzeng, K. J., Su, C. L., Feng, W., and Plane, J. M. C.: Morphology of sporadic E layer retrieved from COSMIC GPS radio occultation measurements: Wind shear theory examination, J. Geophys. Res.-Space, 119, 2117-2136, doi:10.1002/2013JA019437, 2014.

Forbes, J. M.: Tidal and planetary waves, in: The upper Mesosphere and lower Thermosphere, a review of experiment and theory, edited by: Johnson, R. M. and Killen T. L., 67-87, Geophysical Monograph 87, American Geophysical Union, 1995.

Haldoupis, C.: A tutorial review on Sporadic E layers, in: Aeronomy of the Earth's Atmosphere-Ionosphere, chapter 29, 381-394, IAGA Book Series Volume 2, Springer, 2011.

Haldoupis, C.: Midlatitude Sporadic E layers, A typical paradigm of Atmosphere-Ionosphere coupling, Space Sci. Rev., 168, 441-461, doi:10.1007/s11214-011-9786-8, 2012.

Haldoupis, C., Pancheva, D., and Mitchell, N. J.: A study of tidal and planetary wave periodicities present in midlatitude sporadic E layers, J. Geophys. Res., 109, A02302, doi:10.1029/2003JA010253, 2004.

Haldoupis, C., Meek, C., Christakis, N., Pancheva, D., and Bourdillon, A.: Ionogram height-time intensity observations of descending sporadic $E$ layers at mid-latitude, J. Atmos. Sol. Terr. Phy., 68, 539-557, 2006.

Haldoupis, C., Pancheva, D., Singer, W., Meek, C., and MacDougall, J.: An explanation for the seasonal dependence of midlatitude sporadic E layers, J. Geophys. Res., 112, A06315, doi:10.1029/2007JA012322, 2007. 
Holton, J. R.: The dynamic meteorology of the stratosphere and mesosphere, in: Meteorological Monographs, 15, American Meteorological Society, Boston, 218 pp., 1975.

Lindzen, R. S. and Chapman, S.: Atmospheric Tides, Space Sci. Rev., 10, 3-188, 1969.

Mathews, J. D.: Sporadic E: current views and recent progress, J. Atmos. Sol. Terr. Phy., 60, 413-435, 1998.

Mathews, J. D., Machuga, D. W., and Zhou, Q.: Evidence for electrodynamic linkages between spread-F, ion rain, the intermediate layer, and sporadic E: results from observations and simulations, J. Atmos. Sol.-Terr. Phy., 63, 1529-1543, 2001.

Otsuka, Y., Onoma, F., Shiokawa, K., Ogawa, T., Yamamoto, M., and Fukao, S.: Simultaneous observations of nighttime medium-scale traveling ionospheric disturbances and $\mathrm{E}$ region field-aligned irregularities at midlatitude, J. Geophys. Res., 112, A06317, doi:10.1029/2005JA011548, 2007.

Pezzopane, M.: Interpre: A Windows software for semiautomatic scaling of ionospheric parameters from ionograms, Comput. Geosci., 30, 125-130, doi:10.1016/j.cageo.2003.09.009, 2004.
Pietrella, M., Pezzopane, M., and Bianchi, C.: A comparative sporadic-E layer study between two mid-latitude ionospheric stations, Adv. Space Res., 54, 150-160, doi:10.1016/j.asr.2014.03.019, 2014.

Romano, V., Pau, S., Pezzopane, M., Zuccheretti, E., Zolesi, B., De Franceschi, G., and Locatelli, S.: The electronic Space Weather upper atmosphere (eSWua) project at INGV: advancements and state of the art, Ann. Geophys., 26, 345-351, doi:10.5194/angeo26-345-2008, 2008.

Wakai, N., Ohyama, H., and Koizumi, T.: Manual of Ionogram Scaling, 3rd version, Radio Research Laboratory, Ministry of Posts and Telecommunication, Tokyo, 1987.

Whitehead, J. D.: The formation of the sporadic-E layer in the temperate zones, J. Atmos. Terr. Phy., 20, 49-58, 1961.

Whitehead, J. D.: Recent work on mid-latitude and equatorial sporadic-E, J. Atmos. Terr. Phys., 51, 401-424, 1989.

Zuccheretti, E., Tutone, G., Sciacca, U., Bianchi, C., and Arokiasamy, B. J.: The new AIS-INGV digital ionosonde, Ann. Geophys. Italy, 46, 647-659, 2003. 